\title{
Neutrinos Theory Review
}

\author{
M.C.Gonzalez-Garcia* \\ C.N. Yang Institute for Theoretical Physics, \\ State University of New York at Stony Brook, Stony Brook, NY 11794-3840, USA \\ and \\ Institució Catalana de Recerca i Estudis Avançats (ICREA), \\ Departament d'Estructura i Constituents de la Matèria and Institut de Ciencies del Cosmos, \\ Universitat de Barcelona, Diagonal 647, E-08028 Barcelona, Spain \\ E-mail: concha@insti.physics.sunysb.edu
}

In this talk I will review our present knowledge on neutrino masses and mixing trying to emphasize what has been definitively proved. I will also discuss the most important theoretical implications of these results as well as some avenues in connection with collider phenomenology.

36th International Conference on High Energy Physics,

July 4-11, 2012

Melbourne, Australia

${ }^{*}$ Speaker. 


\section{Introduction}

Neutrino physics is a very exciting field at this moment. From the plenary talks by T.Kobayashi [1] and J.Cao [2] as well as from the talks by P. Novella [3], W.Liangjian [4], K. Sakashita [6], G. Barr [5], Y. Hayato [7], L. Ludhova [8] in the neutrino parallel session we have heard about the enormous progress made and being made in the experimental front in current neutrino oscillation experiments from which we have established with high or at least good precision that:

- Atmospheric $v_{\mu}$ and $\bar{v}_{\mu}$ disappear most likely converting to $v_{\tau}$ and $\bar{v}_{\tau}$. The results show an energy and distance dependence perfectly described by oscillations.

- Accelerator $v_{\mu}$ and $\bar{v}_{\mu}$ disappear over distances of $\sim 200$ to $700 \mathrm{Km}$. The energy spectrum of the results show a clear oscillatory behaviour.

- Solar $v_{e}$ convert to $v_{\mu}$ or $v_{\tau}$. The observed energy dependence of the effect is well described by neutrino conversion in the Sun matter according to the MSW effect [13].

- Reactor $\bar{v}_{e}$ disappear over distances of $\sim 200 \mathrm{Km}$. The observed energy spectrum shows an oscillatory behaviour with a wavelength distinct from the one observed in accelerator $v_{\mu}$ disappearance and compatible with the required parameters for MSW conversion in the Sun.

- Some accelerator $v_{\mu}$ appears as $v_{e}$ at distances $\sim 200$ to $700 \mathrm{Km}$.

- Reactor $\bar{v}_{e}$ disappear also over distances of $\sim 1.5 \mathrm{Km}$.

The last two last results are new since the last ICHEP conference and, as we will see, have help us to finalize the determination of the magnitude of all the entries of the leptonic mixing matrix.

All these results imply that neutrinos are massive and there is physics beyond the Standard Model (SM).

The fundamental question opened by these results is what is the underlying BSM theory for neutrino masses. In this talk, however, I will start on the more mundane but difficult approach of the detailed determination of the minimum low energy parametrization required to describe the bulk of data. The last part of the talk is devoted to discuss the implications of these results trying to follow as much as possible and within its limitations a model independent approach. At these times of LHC operation, I finish by commenting on some avenues which could connect the physics of neutrino masses with low energy lepton flavour experiments and collider phenomenology.

\section{The New Minimal Standard Model}

The $\mathrm{SM}$ is a gauge theory based on the gauge symmetry $S U(3)_{\mathrm{C}} \times S U(2)_{\mathrm{L}} \times U(1)_{\mathrm{Y}}$ spontaneously broken to $S U(3)_{\mathrm{C}} \times U(1)_{\mathrm{EM}}$ by the the vacuum expectation value of a Higgs doublet field $\phi$. The SM contains three fermion generations which reside in chiral representations of the gauge group. Right-handed fields are included for charged fermions as they are needed to build the electromagnetic and strong currents. No right-handed neutrino is included in the model since neutrinos are neutral and colourless and therefore the right-handed neutrinos are singlets of the SM group.

In the SM, fermion masses arise from the Yukawa interactions which couple the right-handed fermion singlets to the left-handed fermion doublets and the Higgs doublet. After spontaneous electroweak symmetry breaking (EWSSB) these interactions lead to charged fermion masses but leave the neutrinos massless. No Yukawa interaction can be written that would give a tree level mass to the neutrino because no right-handed neutrino field exists in the model. 
Furthermore, within the SM $G_{\mathrm{SM}}^{\text {global }}=U(1)_{B} \times U(1)_{e} \times U(1)_{\mu} \times U(1)_{\tau}$ is an accidental global symmetry. Here $U(1)_{B}$ is the baryon number symmetry, and $U(1)_{e, \mu, \tau}$ are the three lepton flavor symmetries. Any neutrino mass term which could be built with the particle content of the SM would violate the $U(1)_{L}$ subgroup of $G_{\mathrm{SM}}^{\mathrm{global}}$ and therefore cannot be induced by loop corrections. Also, it cannot be induced by non-perturbative corrections because the $U(1)_{B-L}$ subgroup of $G_{\mathrm{SM}}^{\text {global }}$ is non-anomalous.

It follows then that the SM predicts that neutrinos are strictly massless. Consequently, there is neither mixing nor CP violation in the leptonic sector. Clearly this is in contradiction with the neutrino data summarized in the introduction. So the Standard Model has to be extended at least to include neutrino masses. This minimal extension is what I call The New Minimal Standard Model (NMSM).

The two minimal extensions to give neutrino mass and explain the data are:

- Introduce $v_{R}$ and impose total lepton number $(L)$ conservation so after EWSSB

$$
\mathscr{L}_{D}=\mathscr{L}_{S M}-M_{v} \bar{v}_{L} v_{R}+\text { h.c. }
$$

In this case mass eigenstate neutrinos are Dirac fermions, ie $v^{C} \neq v$.

- Construct a mass term only with the SM left-handed neutrinos by allowing $L$ violation

$$
\mathscr{L}_{M}=\mathscr{L}_{S M}-\frac{1}{2} M_{v} \bar{v}_{L} v_{L}^{c}+\text { h.c. }
$$

In this case the mass eigenstates are Majorana fermions, $v^{C}=v$. Furthermore the Majorana mass term above also breaks the electroweak gauge invariance. In this respect $\mathscr{L}_{M}$ can only be understood as a low energy limit of a complete theory while $\mathscr{L}_{D}$ is formally self-consistent.

Either way, in the NMSM flavour is mixed in the CC interactions of the leptons, and a leptonic mixing matrix appears analogous to the CKM matrix for the quarks. However the discussion of leptonic mixing is complicated by two factors. First the number massive neutrinos $(n)$ is unknown, since there are no constraints on the number of right-handed, SM-singlet, neutrinos. Second, since neutrinos carry neither color nor electromagnetic charge, they could be Majorana fermions. As a consequence the number of new parameters in the model depends on the number of massive neutrino states and on whether they are Dirac or Majorana particles.

In general, if we denote the neutrino mass eigenstates by $v_{i}, i=1,2, \ldots, n$, and the charged lepton mass eigenstates by $l_{i}=(e, \mu, \tau)$, in the mass basis, leptonic $\mathrm{CC}$ interactions are given by

$$
-\mathscr{L}_{\mathrm{CC}}=\frac{g}{\sqrt{2}} \overline{l_{i L}} \gamma^{\mu} U^{i j} v_{j} W_{\mu}^{+}+\text {h.c.. }
$$

Here $U$ is a $3 \times n$ matrix which verifies $U U^{\dagger}=I_{3 \times 3}$ but in general $U^{\dagger} U \neq I_{n \times n}$.

For most of this talk I will be assuming only three massive states. In this case $U$ is a $3 \times 3$ matrix analogous to the CKM matrix for the quarks [14, 15] but due to the Majorana (Dirac) nature of the neutrinos it depends on six (four) independent parameters: three mixing angles and three (one) phases

$$
U=\left(\begin{array}{ccc}
1 & 0 & 0 \\
0 & c_{23} & s_{23} \\
0 & -s_{23} & c_{23}
\end{array}\right) \cdot\left(\begin{array}{ccc}
c_{13} & 0 & s_{13} e^{-i \delta_{\mathrm{CP}}} \\
0 & 1 & 0 \\
-s_{13} e^{i \delta_{\mathrm{CP}}} & 0 & c_{13}
\end{array}\right) \cdot\left(\begin{array}{ccc}
c_{21} & s_{12} & 0 \\
-s_{12} & c_{12} & 0 \\
0 & 0 & 1
\end{array}\right) \cdot\left(\begin{array}{ccc}
e^{i \eta_{1}} & 0 & 0 \\
0 & e^{i \eta_{2}} & 0 \\
0 & 0 & 1
\end{array}\right)
$$


where $c_{i j} \equiv \cos \theta_{i j}$ and $s_{i j} \equiv \sin \theta_{i j}$. In addition to the Dirac-type phase $\delta_{\mathrm{CP}}$, analogous to that of the quark sector, there are two physical phases $\eta_{i}$ associated to the Majorana character of neutrinos.

A consequence of the presence of the leptonic mixing is the possibility of flavour oscillations of the neutrinos [16]. Neutrino oscillations appear because of the misalignment between the interaction neutrino eigenstates and the propagation eigenstates ( which for propagation in vacuum are the mass eigenstates). Thus a neutrino of energy $E$ produced in a $\mathrm{CC}$ interaction with a charged lepton $l_{\alpha}$ can be detected via a CC interaction with a charged lepton $l_{\beta}$ with a probability which presents an oscillatory behaviour, with oscillation lengths given by the phase difference between the different propagation eigenstates - which in the ultrarelativistic limit is $L_{0, i j}^{\mathrm{osc}}=\frac{4 \pi E}{\Delta m_{i j}^{2}}-$ and amplitude that is proportional to elements in the mixing matrix.

It follows that neutrino oscillations are only sensitive to mass squared differences and do not give us information on the absolute value of the masses. Also the Majorana phases do not affect oscillations. As mentioned in the previous section the observed oscillation patterns require two distinctive oscillation wavelengths. Thus there are two possible non-equivalent orderings for the mass eigenvalues, which are conventionally chosen as

$$
\begin{aligned}
& \Delta m_{21}^{2} \ll\left(\Delta m_{32}^{2} \simeq \Delta m_{31}^{2}>0\right) ; \\
& \Delta m_{21}^{2} \ll-\left(\Delta m_{31}^{2} \simeq \Delta m_{32}^{2}<0\right),
\end{aligned}
$$

with $\Delta m_{i j}^{2} \equiv m_{i}^{2}-m_{j}^{2}$. As it is customary we refer to the first option, Eq. (2.5), as Normal ordering (NO), and to the second one, Eq. (2.6), as Inverted ordering (IO); in this form they correspond to the two possible choices of the sign of $\Delta m_{31}^{2}$.

\subsection{The parameters of the NMSM}

In total the 3- $v$ oscillation analysis of the existing data involves six parameters: 2 mass differences (one of which can be positive or negative), 3 mixing angles, and the $\mathrm{CP}$ phase. I summarize in Table 2.1 the different experiments contributing dominantly to the present determination of the different parameters.

\begin{tabular}{l|l|l|} 
Experiment & Dominant & Important \\
\hline Solar Experiments & $\theta_{12}$ & $\Delta m_{21}^{2}, \theta_{13}$ \\
Reactor LBL (KamLAND) & $\Delta m_{21}^{2}$ & $\theta_{12}, \theta_{13}$ \\
Reactor MBL (Daya-Bay, Reno, D-Chooz) & $\theta_{13}$ & $\left|\Delta m_{31,32}^{2}\right|$ \\
Atmospheric Experiments & $\theta_{23}$ & $\left|\Delta m_{31,32}^{2}\right|, \theta_{13}, \delta_{\mathrm{CP}}$ \\
Accelerator LBL $v_{\mu}$ Disapp (Minos) & $\left|\Delta m_{31,32}^{2}\right|$ & $\theta_{23}$ \\
Accelerator LBL $v_{e}$ App (Minos,T2K) & $\delta_{\mathrm{CP}}$ & $\theta_{13}, \theta_{23}$
\end{tabular}

Table 1: Experiments contributing to the present determination of the oscillation parameters.

At present the determination of the leptonic parameters requires global analysis of the data which is in the hands of a few phenomenological groups [17, 18, 19]. The results I summarize here are from Ref. [19]. In Fig1 I show different projections of the allowed six-dimensional parameter space. The best fit values and the derived ranges for the six parameters at the $1 \sigma(3 \sigma)$ level are given in Tab. 2. For each parameter the ranges are obtained after marginalizing with respect to the other parameters. 

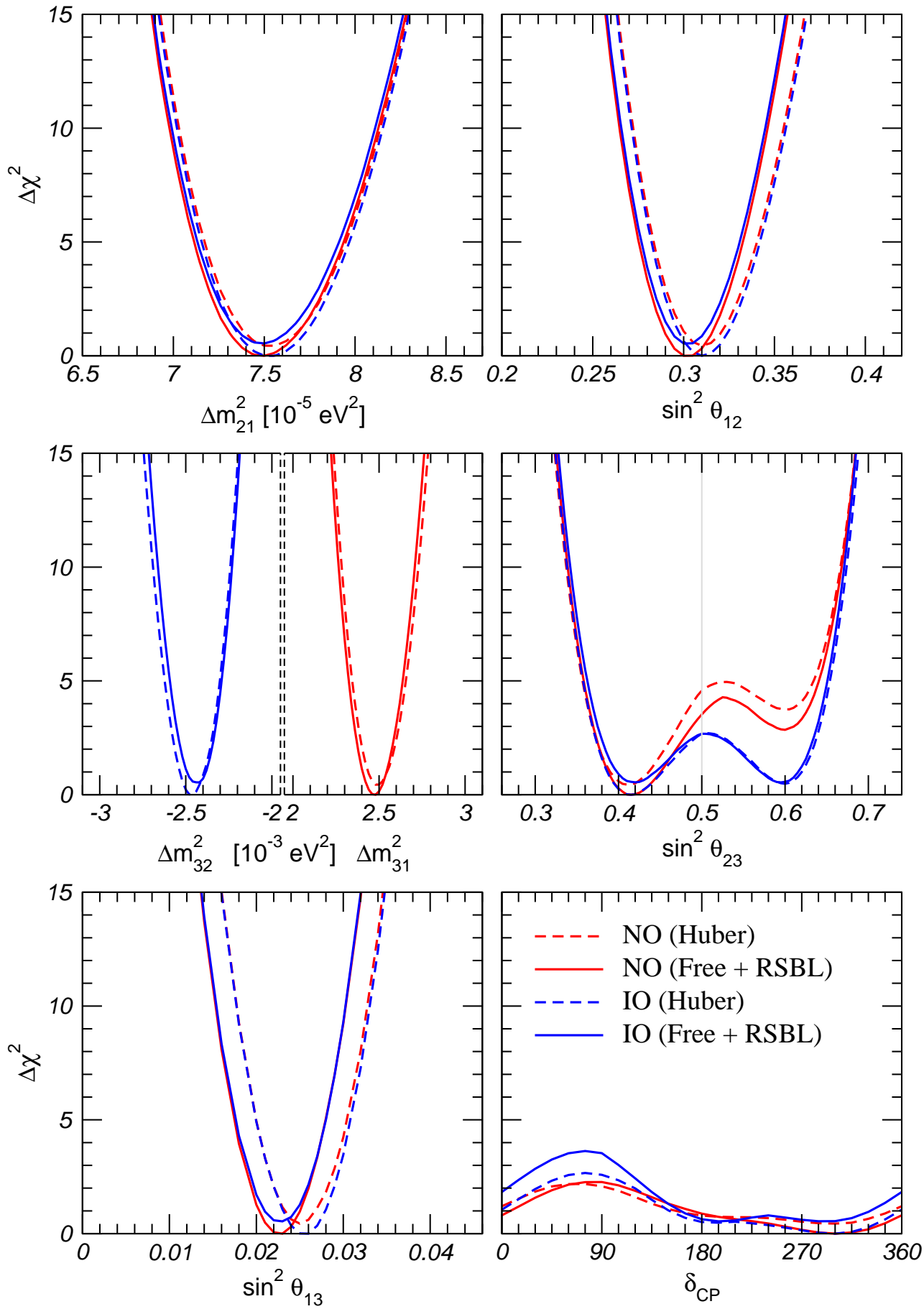

Figure 1: Global 3v oscillation analysis. The red (blue) curves are for Normal (Inverted) Ordering. Results for different assumptions concerning the analysis of data from reactor experiments are shown: for solid curves the normalization of reactor fluxes is left free and data from short-baseline (less than $100 \mathrm{~m}$ ) reactor experiments are included. For dashed curves short-baseline data are not included but reactor fluxes as predicted in [22] are assumed. Note that as atmospheric mass-squared splitting we use $\Delta m_{31}^{2}$ for NO and $\Delta m_{32}^{2}$ for IO. 
The results are shown for two choices of the reactor fluxes. Up to very recently the interpretation of neutrino oscillation searches at nuclear power plants was based on the calculations of the reactor $\bar{v}_{e}$ flux from Ref. [20]. Indeed, the observed rates at all reactor experiments performed so-far at distances $L \lesssim 1 \mathrm{~km}$ (which we label as reactor short-baseline experiments (RSBL) are consistent with these fluxes, therefore setting limits on $\bar{v}_{e}$ disappearance. Over the last two years the flux of $\bar{v}_{e}$ emitted from nuclear power plants has been re-evaluated [21, 22], yielding roughly $3 \%$ higher neutrino fluxes than assumed previously. This might indicate an anomaly in RSBL experiments, which according to the new fluxes observe a slight deficit. Motivated by this situation in Ref. [19] the results are shown for the two limiting assumptions of either taking the predicted fluxes of [22] and ignore the RSBL data (which we label in the figures as "Huber") or to allow for a free normalization of the reactor fluxes and include the RSBL data to determine its possible allowed range (labeled as "Free Fluxes + RSBL").

From these results we conclude that:

1. The present global analysis disfavours $\theta_{13}=0$ with a $\Delta \chi^{2} \approx 100$. This is mostly driven by the new reactor data from Daya Bay, Reno and Double Chooz.

2. An uncertainty on $\theta_{13}$ at the level of $1 \sigma$ remains due to a tension between predicted reactor neutrino fluxes and data from RSBL experiments.

3. Non-maximal $\theta_{23}$ is favoured at the level of $\sim 2 \sigma(\sim 1.5 \sigma)$ for Normal (Inverted) ordering for either choice of the reactor fluxes.

4. The statistical significance of the preference of the fit for the first octant of $\theta_{23}$ is $\leq 1.5 \sigma$ $(\leq 0.9 \sigma)$ for Normal (Inverted) ordering for either choice of the reactor fluxes.

5. When the normalization of reactor fluxes is left free and data from RSBL experiments are included, the absolute best-fit occurs for Normal ordering but the statistical significance of the preference Normal versus Inverted is $\leq 0.7 \sigma$.

6. The best fit occurs for Inverted ordering when RSBL data are not included and reactor fluxes as predicted in [22] are assumed but the statistical significance of the preference Inverted versus Normal is $\leq 0.75 \sigma$.

7. The statistical significance of the effects associated with $\delta_{\mathrm{CP}}$ is $\leq 1.5 \sigma(\leq 1.75 \sigma)$ for Normal (Inverted) ordering.

It is important to stress that in the present global analysis both the sensitivity to the octant of $\theta_{23}$ and to $\delta_{\mathrm{CP}}$ still relies on the observability of sub-dominant oscillation effects in the atmospheric neutrino analysis. Atmospheric neutrino results from Super-Kamiokande collaboration are presented in terms of a large number of data samples and the rates for some of those samples cannot be theoretically predicted (and therefore included in a statistical analysis) without a detailed simulation of the detector, which can only be made by the experimental collaboration itself. Hence, although the results of the analysis performed by the different groups contain the most up-to-date analysis of the atmospheric neutrino data which can be performed outside the collaboration, such analysis have unavoidable limitations. Caution is mandatory when "hints" of such small effects are claimed. 


\begin{tabular}{l|cc|cc}
\hline \hline & \multicolumn{2}{|c|}{ Free Fluxes + RSBL } & \multicolumn{2}{c}{ Huber Fluxes, no RSBL } \\
\hline & bfp $\pm 1 \sigma$ & $3 \sigma$ range & bfp $\pm 1 \sigma$ & $3 \sigma$ range \\
\hline $\sin ^{2} \theta_{12}$ & $0.302_{-0.012}^{+0.013}$ & $0.267 \rightarrow 0.344$ & $0.311_{-0.013}^{+0.013}$ & $0.273 \rightarrow 0.354$ \\
$\theta_{12} /^{\circ}$ & $33.36_{-0.78}^{+0.81}$ & $31.09 \rightarrow 35.89$ & $33.87_{-0.80}^{+0.82}$ & $31.52 \rightarrow 36.49$ \\
$\sin ^{2} \theta_{23}$ & $0.413_{-0.025}^{+0.037} \oplus 0.594_{-0.022}^{+0.021}$ & $0.342 \rightarrow 0.667$ & $0.416_{-0.029}^{+0.036} \oplus 0.600_{-0.026}^{+0.019}$ & $0.341 \rightarrow 0.670$ \\
$\theta_{23} /^{\circ}$ & $40.0_{-1.5}^{+2.1} \oplus 50.4_{-1.3}^{+1.3}$ & $35.8 \rightarrow 54.8$ & $40.1_{-1.6}^{+2.1} \oplus 50.7_{-1.5}^{+1.2}$ & $35.7 \rightarrow 55.0$ \\
$\sin ^{2} \theta_{13}$ & $0.0227_{-0.0024}^{+0.0023}$ & $0.0156 \rightarrow 0.0299$ & $0.0255_{-0.0024}^{+0.0024}$ & $0.0181 \rightarrow 0.0327$ \\
$\theta_{13} /{ }^{\circ}$ & $8.66_{-0.46}^{+0.44}$ & $7.19 \rightarrow 9.96$ & $9.20_{-0.45}^{+0.41}$ & $7.73 \rightarrow 10.42$ \\
$\delta_{\mathrm{CP}} /^{\circ}$ & $300_{-138}^{+66}$ & $0 \rightarrow 360$ & $298_{-145}^{+59}$ & $0 \rightarrow 360$ \\
$\frac{\Delta m_{21}^{2}}{10^{-5} \mathrm{eV}^{2}}$ & $7.50_{-0.19}^{+0.18}$ & $7.00 \rightarrow 8.09$ & $7.51_{-0.15}^{+0.21}$ & $7.04 \rightarrow 8.12$ \\
$\frac{\Delta m_{31}^{2}}{10^{-3} \mathrm{eV}^{2}}(\mathrm{~N})$ & $+2.473_{-0.067}^{+0.070}$ & $+2.276 \rightarrow+2.695$ & $+2.489_{-0.051}^{+0.055}$ & $+2.294 \rightarrow+2.715$ \\
$\frac{\Delta m_{32}^{2}}{10^{-3} \mathrm{eV}^{2}}(\mathrm{I})$ & $-2.427_{-0.065}^{+0.042}$ & $-2.649 \rightarrow-2.242$ & $-2.468_{-0.065}^{+0.073}$ & $-2.678 \rightarrow-2.252$ \\
\hline \hline
\end{tabular}

Table 2: Three-flavour oscillation parameters from our fit to global data after the Neutrino 2012 conference. For "Free Fluxes + RSBL" reactor fluxes have been left free in the fit and short baseline reactor data (RSBL) with $L \lesssim 100$ m are included; for "Huber Fluxes, no RSBL" the flux prediction from [22] are adopted and RSBL data are not used in the fit.

From the global $\chi^{2}$ one can derive the following $3 \sigma$ CL ranges on the magnitude of the elements of the leptonic mixing matrix

$$
|U|=\left(\begin{array}{lll}
0.795 \rightarrow 0.846 & 0.513 \rightarrow 0.585 & 0.126 \rightarrow 0.178 \\
0.205 \rightarrow 0.543 & 0.416 \rightarrow 0.730 & 0.579 \rightarrow 0.808 \\
0.215 \rightarrow 0.548 & 0.409 \rightarrow 0.725 & 0.567 \rightarrow 0.800
\end{array}\right)
$$

By construction the derived limits in Eq. (2.7) are obtained under the assumption of the matrix $U$ being unitary.

\subsection{Neutrino Mass Scale: Laboratory-Cosmology Connection}

Oscillation experiments provide information on $\Delta m_{i j}^{2}$, and on the leptonic mixing angles, $U_{i j}$. But they are insensitive to the absolute mass scale for the neutrinos. Of course, the results of an oscillation experiment do provide a lower bound on the heavier mass in $\Delta m_{i j}^{2},\left|m_{i}\right| \geq \sqrt{\Delta m_{i j}^{2}}$ for $\Delta m_{i j}^{2}>0$. But there is no upper bound on this mass. In particular, the corresponding neutrinos could be approximately degenerate at a mass scale that is much higher than $\sqrt{\Delta m_{i j}^{2}}$. Moreover, there is neither upper nor lower bound on the lighter mass $m_{j}$.

Information on the neutrino masses, rather than mass differences, can be extracted from kinematic studies of reactions in which a neutrino or an anti-neutrino is involved. In the presence of mixing the most relevant constraint comes from Tritium beta decay ${ }^{3} \mathrm{H} \rightarrow{ }^{3} \mathrm{He}+\mathrm{e}^{-}+\bar{v}_{\mathrm{e}}$ which, within the present and expected experimental accuracy, can limit the combination

$$
m_{\beta}=\sum_{i} m_{i}\left|U_{e i}\right|^{2}
$$


The present bound is $m_{\beta} \leq 2.2 \mathrm{eV}$ at $95 \%$ CL [23] which is expected to be superseded soon by KATRIT [9]-

Direct information on neutrino masses can also be obtained from neutrinoless double beta decay $(A, Z) \rightarrow(A, Z+2)+e^{-}+e^{-}$. The rate of this process is proportional to the effective Majorana mass of $v_{e}$,

$$
m_{e e}=\left|\sum_{i} m_{i} U_{e i}^{2}\right|
$$

which, unlike Eq. (2.8), depends also on the three CP violating phases. Notice that in order to induce the $2 \beta 0 v$ decay, $v$ 's must be Majorana particles. For years the strongest bound from $2 \beta 0 v$ decay was $m_{e e}<0.34 \mathrm{eV}$ at $90 \% \mathrm{CL}$ [24] (which taking into account systematic errors related to nuclear matrix elements may be weakened by a factor of about 3 ). As we heard in this conference this range of masses is started to be tested also by running experiments like EXO [10] and KamLAND-ZEN [11]. A series of new experiments is planned with sensitivity of up to $m_{e e} \sim 0.01$ $\mathrm{eV}$ [12].

Neutrinos, like any other particles, contribute to the total energy density of the Universe. Furthermore within what we presently know of their masses neutrinos are relativistic through most of the evolution of the Universe and they are very weakly interacting which means that they decoupled early in cosmic history. Depending on their exact masses they can impact the CMB spectra, in particular by altering the value of the redshift for matter-radiation equality. More importantly, their free streaming suppresses the growth of structures on scales smaller than the horizon at the time when they become non-relativistic and therefore affects the matter power spectrum which is probed from surveys of the LSS distribution. Within their present precision, cosmological observations are sensitive to neutrinos only via their contribution to the energy density in our Universe, $\Omega_{v} h^{2}=\sum_{i} m_{i} /(94 \mathrm{eV})$ Therefore cosmological data mostly gives information on the sum of the neutrino masses and has very little to say on their mixing structure and on the ordering of the mass states.

Correlated information on the three probes of neutrino masses can be obtained by mapping the results from the global analysis of oscillations presented in the previous section in combination with the information from global analysis of cosmological data [25]. I show in Fig. 2 the present status of this exercise from Ref.[26]

\section{Some Implications}

The determined ranges of neutrino masses and leptonic mixing raise two main questions:

- Why are neutrinos so light?, which is directly related to issue of the origin of neutrino mass.

- Why is lepton mixing so different from quark mixing?, which is related to the flavour puzzle.

The relevance of the new determination on $\theta_{13}$ in the answer of this second question is illustrated in Fig.3 where I show the compilation in Ref.[27] (taken from [28]) of the predictions of the expected values of $\theta_{13}$ is 63 types of flavour models. As seen from the figure only about $10 \%$ of the models survived the test. Among the successful are the proposal based on flavour anarchy of Ref.[29] which predicted relatively large values of $\theta_{13}$ but still compatible with the measured values with about $40 \%$ probability. The observation of a possible quark-lepton complementarity 


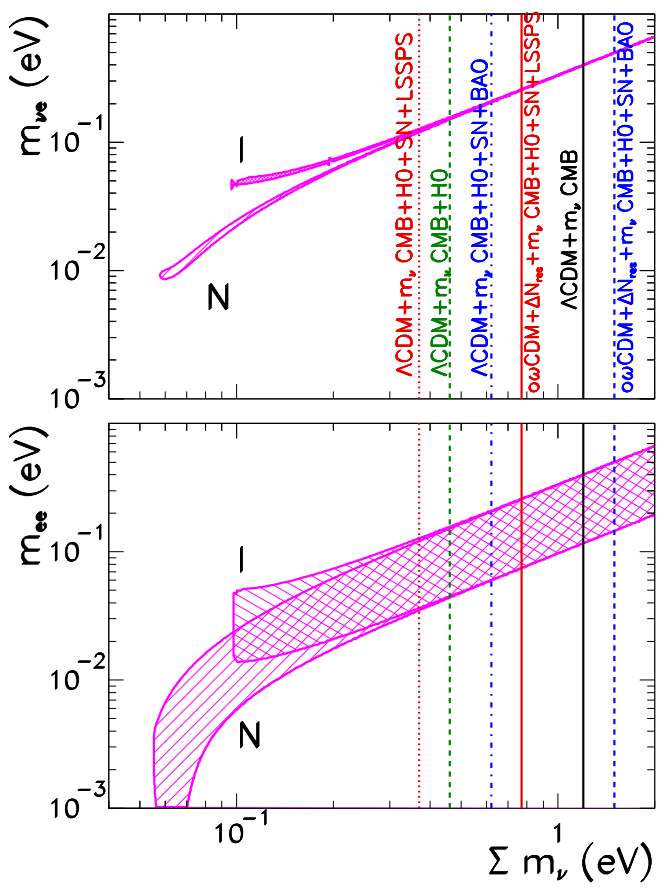

Figure 2: $95 \%$ allowed regions (for 2 dof) in the planes $\left(m_{v_{e}}, \sum m_{v}\right)$ and $\left(m_{e e}, \sum m_{v}\right)$ from the global analysis of oscillation data (full regions). We also show superimposed the $95 \%$ upper bounds on $\sum m_{v}$ from cosmological constraints for the different analysis presented in Ref.[26].

[30] based on the empirical relation $\theta_{C}+\theta_{12}=40^{\circ}$ lead to the prediction $\left|U_{e 3}\right|=\tan \theta_{C}\left|U_{\mu 3}\right| \rightarrow$ $\sin ^{2} \theta_{13}=0.026$ which is in perfect agreement with the present determination.

Among those which did not survive the test of the precise determination of the mixing parameters were the models predicting bimaximal mixing $\left(\theta_{12}=\theta_{23}=45^{\circ}, \theta_{13}=0\right)$ [31], tri-bimaxmial mixing $\left(\theta_{12}=35.2^{\circ} \theta_{23}=45^{\circ}, \theta_{13}=0\right)[32]$, and the golden ratio $\left(\theta_{12}=31.7^{\circ} \theta_{23}=45^{\circ}, \theta_{13}=0\right)$ [33]. Generically these structures appear in models with flavour symmetries with the smallest symmetry groups $A_{4}, S_{4}$ and $A_{5}$. Consequently either the group has to be enlarged, or corrections to the mixing have to be obtained from other sectors, like, for example, from the charge lepton mixings in GUTs inspired models [28]. Generically these attempts lead to new sum rules relating the leptonic flavour parameters among themselves and with those of quarks. Relations which can be testable with enough experimental precision.

\subsection{Neutrino Masses and Effective Lagrangians}

There are many good reasons to think that the SM is not a complete picture of Nature and some new physics (NP) is expected to appear at higher energies. In this case the SM is an effective low energy theory valid up to the scale $\Lambda_{\mathrm{NP}}$ which characterizes the NP. In this approach, the gauge group, the fermionic spectrum, and the pattern of spontaneous symmetry breaking are still valid ingredients to describe Nature at energies $E \ll \Lambda_{\mathrm{NP}}$. The difference between the SM as a complete description of Nature and as a low energy effective theory is that in the latter case we must consider 


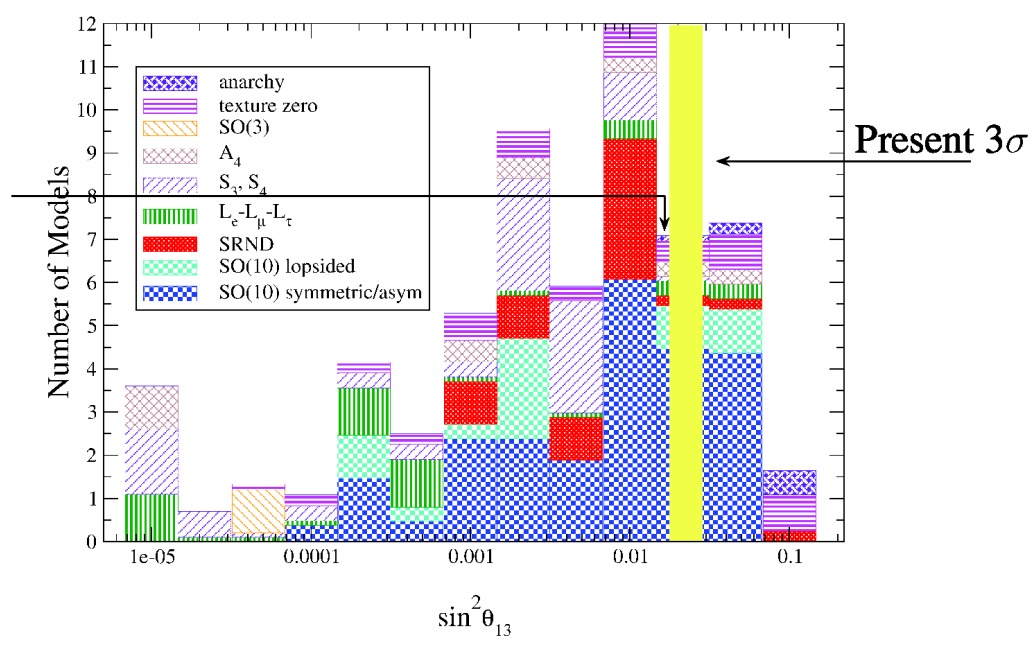

Figure 3: Compilation in Ref.[27] of the prediction of the value of $\theta_{13}$ in several flavour models compared with the present determination.

also non-renormalizable $(\operatorname{dim}>4)$ terms whose effect will be suppressed by powers $1 / \Lambda_{\mathrm{NP}}^{\mathrm{dim}-4}$. In this approach the largest effects at low energy are expected to come from $\operatorname{dim}=5$ operators.

There is a single set of dimension-five terms that is made of SM fields and is consistent with the gauge symmetry given by

$$
\frac{c_{5 \alpha \beta}}{\Lambda_{\mathrm{NP}}} \mathscr{O}_{5}=\frac{c_{5 \alpha \beta}}{\Lambda_{\mathrm{NP}}}\left(\bar{L}_{L \alpha} \tilde{\phi}\right)\left(\phi^{+} L_{L \beta}^{C}\right)+\text { h.c. },
$$

which violates total lepton number by two units and leads, upon EWSB, to neutrino masses:

$$
\left(M_{v}\right)_{\alpha \beta}=c_{5 \alpha \beta} \frac{v^{2}}{\Lambda_{\mathrm{NP}}} \text {. }
$$

This is a Majorana mass term.

Eq. (3.2) arises in a generic extension of the SM which means that neutrino masses are very likely to appear if there is NP. Furthermore from Eq. (3.2) we find that the scale of neutrino masses is suppressed by $v / \Lambda_{\mathrm{NP}}$ when compared to the scale of charged fermion masses providing an explanation not only for the existence of neutrino masses but also for their smallness. Finally, Eq. (3.2) breaks not only total lepton number but also the lepton flavor symmetry. Thus we should expect lepton mixing and $\mathrm{CP}$ violation.

Given the relation (3.2), $m_{v} \sim v^{2} / \Lambda_{\mathrm{NP}}$, it is straightforward to use measured neutrino masses to estimate the scale of NP that is relevant to their generation. In particular, if there is no quasidegeneracy in the neutrino masses, the heaviest of the active neutrino masses can be estimated, $m_{h}=m_{3} \sim \sqrt{\Delta m_{31}^{2}} \approx 0.05 \mathrm{eV}$ (in the case of inverted hierarchy the implied scale is $m_{h}=m_{2} \sim$ $\sqrt{\left|\Delta m_{31}^{2}\right|} \approx 0.05 \mathrm{eV}$ ). It follows that the scale in the non-renormalizable term (3.1) is given by

$$
\Lambda_{\mathrm{NP}} \sim v^{2} / m_{h} \approx 10^{15} \mathrm{GeV}
$$

We should clarify two points regarding Eq. (3.3): 

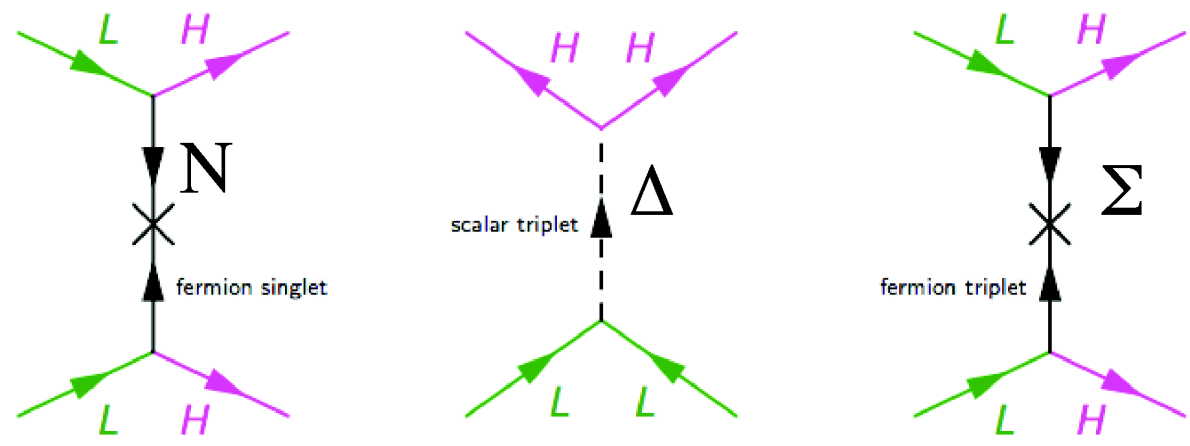

Figure 4: Tree level diagrams for the Type-I,II and III see-saw, leading to the dim-5 operator for neutrino mass after integrating out the intermediate state

1. There could be some level of degeneracy between the neutrino masses that are relevant to the atmospheric neutrino oscillations. In such a case Eq. (3.3) becomes an upper bound on the scale of NP.

2. It could be that the $c_{5 \alpha \beta}$ couplings of Eq. (3.1) are much smaller than one. In such a case, again, Eq. (3.3) becomes an upper bound on the scale of NP.

Of course, neutrinos could be conventional Dirac particles described as in Eq. (2.1) and we would be left in the darkness on the reason of the smallness of the neutrino mass.

In simple renormalizable realizations of NP this dimension-5 operator can be generated by the tree-level exchange of three types of new particles (see Fig.4):

- Type-I [34] and Type-III [36] see-saw : One adds at least two fermionic singlets (Type-I) or triplets (Type-III) of mass $M$ and Yukawa couplings $\lambda$. The neutrino masses are as Eq. (3.2) with $\Lambda_{\mathrm{NP}}=M$ and $c_{5} \sim \lambda^{2}$.

- Type-II see-saw [35]: One adds an $S U(2)_{L}$ Higgs triplet $\Delta$ of mass $M$ which couples to the SM $S U(2)_{L}$ leptons with coupling $f$, with a neutral component and scalar doublet-triplet mixing $\mu$ term in the scalar potential. The neutrino masses are as Eq. (3.2) with $\Lambda_{\mathrm{NP}}=M^{2} / \mu$ and $c_{5} \sim f$.

One may notice that even in these particularly simple forms of NP, the full theory contains very different high-energy particle contents but they lead to the same low energy operator $\mathscr{O}_{5}$, which contains only 9 parameters which is everything we can measure at the neutrino oscillation experiments. This simple example illustrates the limitation of the "bottom-up" approach in deriving model independent implications of the presently observed neutrino masses and mixing. This is the challenge of performing measurements at a much lower scale than that of the NP.

Alternatively one can go "top-down" by studying the low energy effective neutrino masses and mixing induced by specific high energy models as sketched in the discussion about flavour models above.

The bottom line of this discussion is that in order to advance further in the understanding of the dynamics underlying neutrino masses in a model independent approach we need more (and more precise) data. Furthermore synergy among different types of observations such as charge lepton flavour experiments and collider experiments are probably going to be fundamental in this advance. In this respect I will finish by discussing a possible framework in which this connection between neutrino physics and collider signatures arises. 
Generically, at low energies the Lagrangian of the full theory can be expanded as

$$
\mathscr{L}=\mathscr{L}_{S M}+\frac{c_{5}}{\Lambda_{L N}} \mathscr{O}_{5}+\sum_{i} \frac{c_{6, i}}{\Lambda_{F L}^{2}} \mathscr{O}_{6, i}+\ldots
$$

where $\mathscr{O}_{5}$ is Weinberg's operator responsible for neutrino masses given above, and $\mathscr{O}_{6, i}$ are flavourchanging, but lepton number conserving, dimension-6 operators. In writing Eq.(3.4) we have explicitly denoted $\Lambda_{L N}$ as the NP scale for lepton number breaking and $\Lambda_{F L}$ the NP scale for lepton flavour breaking. In this context attractive testable scenarios are those for which it is possible to relate the mass of the new states $M \sim \Lambda_{F L} \sim \mathscr{O}$ (TeV) but still keep $\Lambda_{L N} \gg \Lambda_{F L}$ to explain the smallness of the neutrino mass.

Furthermore to relate the flavour structure of the signals at collider, or low energy charged lepton flavour experiments with that derived from the neutrino sector one would need some connection between the coefficients $c_{5}$ and $c_{6}$. This is precisely provided by the assumption of minimal lepton flavour violation (MLFV) [38, 39, 37, 40] of the NP. Indeed these conditions are automatically fulfilled by the simplest Type-II see-saw model if a light double-triplet mixing $\mu$ is assumed. For LHC phenomenology this leads to the interesting possibility studied in detail in Refs. [41, 42] of the production of the triplet scalar states with all their decay modes determined by the neutrino mass parameters. From the theoretical side, one drawback of such a scenario is that it is difficult to keep such a low $\mu$ stable if generated by spontaneous breaking of lepton number. The possibility of constructing MLFV scenarios of Type-I and Type-III see-saws was explored in Ref.[37]. The observability of the corresponding signals at LHC was studied in Ref.[43].

\section{Conclusion}

Neutrino physics is a very exciting field which is at the moment experimentally driven. An enormous experimental effort has been devoted in the last years to prove beyond doubt the presence of neutrino masses and mixing. In particular this year has seen the determination of the third mixing angle in the leptonic mixing matrix $\theta_{13}$.

Neutrino masses imply physics beyond the Standard Model. Determining the parameters of the neutrino mass matrix is providing fundamental information to understand the dynamics at the new physics scale. However even at the end of the existing neutrino programs, we will still be far from reaching this goal. Further advance requires a new generation of neutrino experiments. Furthermore, only with neutrino experiments it is impossible to completely determine the new physics dynamics. In the era of LHC one can only hope for signatures of the new dynamics which will help us to finally establish the new standard model.

Acknowledgments This work is supported by USA-NSF grant PHY-09-6739, by CUR Generalitat de Catalunya grant 2009SGR502 by MICINN FPA2010-20807 and consolider-ingenio 2010 program grant CUP (CSD-2008-0037) and by EU grant FP7 ITN INVISIBLES (Marie Curie Actions PITN-GA-2011-289442).

\section{References}

[1] T. Kobayashi in these proceedings. 
[2] J. Cao in these proceedings.

[3] P. Novella in these proceedings.

[4] W. Liangjian in these proceedings.

[5] G. Barr in these proceedings.

[6] K. Sakashita in these proceedings.

[7] Y. Hayato in these proceedings.

[8] L. Ludhova in these proceedings.

[9] V. Hannen in these proceedings.

[10] P.C Rowson in these proceedings.

[11] S. Yamada in these proceedings

[12] See talks by K. Lang, B. Majorovits, S. Di Domizio, in these proceedings.

[13] L. Wolfenstein, Phys. Rev. D 17, 2369 (1978); S.P. Mikheyev, and A.Y. Smirnov, Yad. Fiz. 42, 1441 (1985) [Sov. J. Nucl. Phys. 42, 913].

[14] Z. Maki, M. Nakagawa, and S. Sakata, Prog. Theor. Phys. 28 (1962) 870-880.

[15] M. Kobayashi and T. Maskawa, Prog. Theor. Phys. 49 (1973) 652-657.

[16] B. Pontecorvo, J. Exptl. Theoret. Phys. 33, 549 (1957).

[17] G. Fogli, E. Lisi, A. Marrone, D. Montanino, A. Palazzo, et al., Phys.Rev. D86 (2012) 013012,

[18] D. Forero, M. Tortola, and J. Valle, Phys.Rev. D86 (2012) 073012,

[19] M. C. Gonzalez-Garcia, M. Maltoni, J. Salvado and T. Schwetz, arXiv:1209.3023 [hep-ph]; NuFit webpage, www. nu-fit.org.

[20] K. Schreckenbach, G. Colvin, W. Gelletly, and F. Von Feilitzsch, Phys.Lett. B160 (1985) 325-330.

[21] T. Mueller, D. Lhuillier, M. Fallot, A. Letourneau, S. Cormon, et al., Phys.Rev. C83 (2011) 054615.

[22] P. Huber, Phys.Rev. C84 (2011) 024617.

[23] J. Bonn, et al., Nucl. Phys. Proc. Suppl. 91, 273 (2001); V.M. Lobashev, et al., Nucl. Phys. Proc. Suppl. 91, 280 (2001).

[24] H.V. Klapdor-Kleingrothaus, H.V., Eur. Phys. J. A 12147 (2001).

[25] G. L. Fogli et al., Phys. Rev. D 70 (2004) 113003,

[26] M. C. Gonzalez-Garcia, M. Maltoni and J. Salvado, JHEP 1008, 117 (2010)

[27] C. H. Albright and M. -C. Chen, Phys. Rev. D 74, 113006 (2006) [hep-ph/0608137].

[28] S. Antusch in these proceedings.

[29] L. J. Hall, H. Murayama and N. Weiner, Phys. Rev. Lett. 84, 2572 (2000) [hep-ph/9911341].

[30] H. Minakata and A. Y. .Smirnov, Phys. Rev. D 70, 073009 (2004) [hep-ph/0405088].

[31] V. D. Barger, S. Pakvasa, T. J. Weiler and K. Whisnant, Phys. Lett. B 437, 107 (1998) [hep-ph/9806387].

[32] P. F. Harrison, D. H. Perkins and W. G. Scott, Phys. Lett. B 530, 167 (2002) [hep-ph/0202074]. 
[33] Y. Kajiyama, M. Raidal and A. Strumia, Phys. Rev. D 76, 117301 (2007) [arXiv:0705.4559 [hep-ph]]; G. -J. Ding, L. L. Everett and A. J. Stuart, Nucl. Phys. B 857, 219 (2012) [arXiv:1110.1688 [hep-ph]];F. Feruglio and A. Paris, JHEP 1103, 101 (2011) [arXiv:1101.0393 [hep-ph]].

[34] P. Minkowski, Phys. Lett. B 67 (1977) 421; T. Yanagida, in Proceedings of the Workshop on the Unified Theory and the Baryon Number in the Universe, eds. O. Sawada et al., (KEK Report 79-18, Tsukuba, 1979), p. 95; M. Gell-Mann, P. Ramond and R. Slansky, in Supergravity, eds. P. van Nieuwenhuizen et al., (North-Holland, 1979), p. 315; R. N. Mohapatra and G. Senjanović, Phys. Rev. Lett. 44 (1980) 912.

[35] W. Konetschny and W. Kummer, Phys. Lett. B 70 (1977) 433; T. P. Cheng and L. F. Li, Phys. Rev. D 22 (1980) 2860; G. Lazarides, Q. Shafi and C. Wetterich, Nucl. Phys. B 181 (1981) 287; J. Schechter and J. W. F. Valle, Phys. Rev. D 22 (1980) 2227; R. N. Mohapatra and G. Senjanović, Phys. Rev. D 23 (1981) 165.

[36] R. Foot, H. Lew, X. G. He and G. C. Joshi, Z. Phys. C 44 (1989) 441.

[37] M. B. Gavela, T. Hambye, D. Hernandez and P. Hernandez, JHEP 0909 (2009) 038 [arXiv:0906.1461 [hep-ph]].

[38] V. Cirigliano, B. Grinstein, G. Isidori and M.B. Wise, Nucl. Phys. B 728 (2005) 121.

[39] S. Davidson and F. Palorini, Phys. Lett. B 642 (2006) 72 [arXiv:hep-ph/0607329].

[40] R. Alonso, G. Isidori, L. Merlo, L. A. Munoz, E. Nardi, JHEP 1106 (2011) 037. [arXiv:1103.5461 [hep-ph]].

[41] P. Fileviez Perez, T. Han, G. -y. Huang, T. Li, K. Wang, Phys. Rev. D78, 015018 (2008). [arXiv:0805.3536 [hep-ph]].

[42] J. Garayoa and T. Schwetz, JHEP 0803, 009 (2008) [arXiv:0712.1453 [hep-ph]].

[43] O. J. P. Eboli, J. Gonzalez-Fraile and M. C. Gonzalez-Garcia, JHEP 1112, 009 (2011) [arXiv:1108.0661 [hep-ph]]. 